\title{
Identification of atrophy of the subgenual anterior cingulate cortex, in particular the subcallosal area, as an effective auxiliary means of diagnosis for major depressive disorder
}

\author{
This article was published in the following Dove Press journal: \\ International Journal of General Medicine \\ 2 August 2012 \\ Number of times this article has been viewed
}

\author{
Akira Niida' \\ Richi Niida ${ }^{2}$ \\ Hiroshi Matsuda ${ }^{3}$ \\ Takashi Inada ${ }^{4}$ \\ Makoto Motomura ${ }^{5}$ \\ Akihiko Uechi ${ }^{6}$ \\ 'Department of Radiology, Nanbu \\ Hospital, Itoman City, Okinawa, \\ ${ }^{2}$ Department of Psychiatry, Nanto \\ Clinic, Urasoe City, Okinawa, \\ ${ }^{3}$ Department of Nuclear Medicine, \\ Saitama Medical University \\ International Medical Center, \\ Hidaka City, Saitama, ${ }^{4}$ Department \\ of Psychiatry, Kai Clinic, Naha \\ City, Okinawa, ${ }^{5}$ Department of \\ Human Sciences, University of the \\ Ryukyu, Nakagami County, Okinawa, \\ ${ }^{6}$ Cognitive Neuroscience Research \\ Project, Kansai Gaidai University, \\ Hirakata City, Osaka, Japan
}

\begin{abstract}
Background: Despite being a very common psychiatric disorder, physicians often have difficulty making a diagnosis of major depressive disorder (MDD) because, without established diagnostic criteria, they have to depend on interviews with patients and observation to assess psychiatric symptoms. However, previous researchers have reported that magnetic resonance imaging (MRI) scans identify morphological changes in the brains of patients with MDD, which inspired us to hypothesize that assessment of local changes in the brain using voxel-based morphometry would serve as an auxiliary diagnostic method for MDD. Therefore, we focused on the VSRAD ${ }^{\circledR}$ plus (voxel-based specific regional analysis system for Alzheimer’s disease), a diagnostic support system for use in early Alzheimer's disease, which allowed us to identify regional atrophy in the brain easily based on images obtained from MRI scans.
\end{abstract}

Methods: The subjects were 75 patients with MDD, 15 with bipolar disorder, and 30 healthy subjects, aged 54-82 years. First, 1.5 T MRI equipment was used to scan three-dimensional $\mathrm{T}_{1}$-weighted images for the individual subjects, and the imaged data were analyzed by VSRAD advance (voxel-based morphometric software developed for diagnosis of early Alzheimer's disease). The efficacy of the equipment for diagnosis of MDD was evaluated based on the distribution of atrophy in the subgenual anterior cingulate cortex (sACC) on the z-score map obtained.

Results: No significant difference in atrophy was noted between the left and right sACCs. The VSRAD advance used in the present study was more effective than the VSRAD plus for diagnosis of MDD, with a sensitivity of $90.7 \%$, specificity of $86.7 \%$, accuracy of $89.5 \%$, a positive predictive value of $94.4 \%$, and a negative predictive value of $78.8 \%$. In particular, atrophy was observed in the subcallosal area of the sACC.

Conclusion: The identification of atrophy in the sACC, in particular of the subcallosal area, with the use of updated voxel-based morphometric software proved to be effective as an auxiliary diagnostic method for MDD.

Keywords: major depressive disorder, magnetic resonance imaging, subgenual anterior cingulate cortex, voxel-based morphometry, VSRAD

\section{Introduction}

Major depressive disorder (MDD) is a very common psychiatric disorder, with a lifetime prevalence of approximately $20 \%$. When making a diagnosis of MDD, psychiatrists usually interview patients or observe them to assess their psychiatric symptoms although, without established visual diagnostic criteria, they often have difficulty.
Nanbu

Itoman City, Okinawa 901-0362,

Japan

Tel +81989940501

Fax +81989940525

Email aniida@nirai.ne.jp 
In recent years, studies have been conducted involving the local morphology and function of the brain using magnetic resonance imaging (MRI), single-photon emission computed tomography, and positron emission tomography, as well as genetic research. However, few studies have been performed in the clinical setting, and there is no established method for auxiliary diagnosis of MDD.

We hypothesized that assessment of local morphological changes in the brain might serve as an auxiliary diagnostic method for MDD. Using a voxel-based specific regional analysis system for Alzheimer's disease (VSRAD ${ }^{\circledR}$ plus, Eisai Co, Ltd, Tokyo, Japan), which was developed based on the voxel-based morphometry method and is now freely available, ${ }^{1-4}$ we investigated the subgenual anterior cingulate cortex (sACC), amygdala, hippocampus, and prefrontal area, reported to be associated with MDD in earlier studies, ${ }^{5-23}$ and made the following observations. Atrophy of the sACC was noted in 65 of $71 \mathrm{MDD}$ patients, and the prevalence was higher than that observed in the hippocampus, amygdala, and prefrontal area (in 35, 21, and 20 patients, respectively). The sensitivity, specificity, and accuracy of the diagnosis of MDD based on the presence or absence of atrophy in the sACC was $93 \%, 85 \%$, and 90\%, respectively. ${ }^{4}$ Accordingly, we suggested that identification of atrophy in the sACC (including a part of the orbitofrontal area) would be useful for accurate auxiliary diagnosis of MDD.

In the present study, using VSRAD advance updated by incorporating statistical parametric mapping (SPM8, Wellcome Department of Imaging Neuroscience, London, UK) and diffeomorphic anatomic registration through an exponentiated lie algebra (DARTEL, Wellcome Department of Imaging Neuroscience), ${ }^{24}$ we investigated the usefulness of identifying the presence or absence of atrophy in the sACC for diagnosis of MDD.

\section{Materials and methods}

The study was conducted with the approval of the hospital ethics committee. The purpose of the study was explained to all subjects verbally and in written form, and their informed consent was obtained.

The subjects comprised 120 examinees at the pshychiatric clinics aged 54-82 years. This age range was indicated for VSRAD advance because the database included in the software program contained data from healthy volunteers in this age range. The subjects were divided into three groups. One group comprised 75 patients with MDD diagnosed by a psychiatrist based on the Diagnostic and Statistical Manual of Mental Disorders-Fourth Edition (DSM-IV), and included nine males and 66 females of mean age $66.3 \pm 7.6$ years. Patients with cognitive dysfunction were excluded. The second group comprised 15 patients with bipolar disorder diagnosed according to the DSM-IV, and included seven males and eight females of mean age $63.1 \pm 4.8$ years. The third group comprised 30 healthy subjects with no past medical history of MDD who underwent MRI to exclude organic disease, and included six males and 24 females of mean age $65.8 \pm 5.4$ years. The gender ratio was significantly different between the groups, but no significant difference was noted for subject age. Disease severity based on the Hamilton Rating Scale for Depression was 12-31 (mean 19.3 \pm 3.4 ) in the 75 patients in the MDD group; this score was lower than 13 in one patient, $14-18$ in $42,19-22$ in 13 , and $\geq 23$ in 19 . The duration of illness was $0.9-10$ (mean $4.9 \pm 2.7$ ) years; $\leq 0.5$ years in 0 patients, $0.6-1$ year in $10,1.1-3$ years in $22,3.1-6$ years in 19 , and $\geq 6.1$ years in 24 . The duration of treatment was $0.5-9$ (mean $3.4 \pm 2.5$ ) years, $\leq 0.5$ years in seven patients, $0.6-1$ year in $18,1.1-3$ years in $19,3.1-6$ years in 18 , and $\geq 6.1$ years in 13 patients. In the bipolar disorder group comprising 15 patients, the mean duration of disease was $11.5 \pm 3.2$ years, which was slightly longer than that in the MDD group. The mean duration of treatment was $6.2 \pm 4.0$ years, showing no significant difference from that in the MDD group (Table 1). Only mild nonmorbid lesions in the white matter were noted on $\mathrm{T}_{2}$-weighted imaging, and no cases of cerebral infarction were detected.

All the subjects were scanned using a $1.5 \mathrm{~T}$ Achieva Nova MRI system (Philips Healthcare, Eindhoven, The Netherlands) and a $T_{1}$-weighted fast field echo sequence was used (field of view, $240 \mathrm{~mm}$; matrix, $256 \times 256$; repetition time, $9.3 \mathrm{msec}$; echo time, $4.3 \mathrm{msec}$; flip angle, $10^{\circ}$; slice thickness, $2 \mathrm{~mm}$, with $1 \mathrm{~mm}$ slices superimposed). A total of $160 \mathrm{~T}_{1}$-weighted three-dimensional sagittal sections of the brains were acquired. The acquisition time was 3 minutes and 35 seconds. The Digital Imaging and Communication in Medicine data obtained were automatically analyzed on a PC using the VSRAD advance program. First, equalization of voxel sizes and linear and nonlinear transformations were performed. Next, images of gray matter, white matter, and cerebrospinal fluid were separated, and the gray matter images were standardized and smoothed onto templates using DARTEL. Finally, using the z-score analysis method, comparative statistical analysis of the voxels was performed for healthy control and subject databases:

$$
\begin{aligned}
\mathrm{z} \text {-score }= & ([\text { control mean }]-[\text { individual value }]) / \\
& (\text { control standard deviation })
\end{aligned}
$$


Table I Clinical characteristics of the total sample $(n=120)$

\begin{tabular}{|c|c|c|c|c|c|c|c|c|}
\hline \multirow[t]{2}{*}{ Characteristics } & \multicolumn{3}{|c|}{ Mean (SD) } & \multirow[t]{2}{*}{$H$} & \multirow[t]{2}{*}{$U$} & \multirow[t]{2}{*}{$X^{2}$} & \multirow[t]{2}{*}{$d f$} & \multirow[t]{2}{*}{$P$ value } \\
\hline & MDD & BD & $\mathrm{HC}$ & & & & & \\
\hline Sample, no & 75 & 15 & 30 & & & & & \\
\hline \multicolumn{9}{|l|}{ Sex, no } \\
\hline Male & 9 & 7 & 6 & & & 10.11 & 2 & 0.006 \\
\hline Female & 66 & 8 & 24 & & & & & \\
\hline Age, y & $66.3(7.6)$ & $63.1(4.8)$ & $65.8(5.4)$ & 2.13 & & & 2 & 0.34 \\
\hline HAMD, point & $19.3(3.4)$ & & & & & & & \\
\hline-13 & 1 & & & & & & & \\
\hline $14-18$ & 42 & & & & & & & \\
\hline $19-22$ & 13 & & & & & & & \\
\hline $23-$ & 19 & & & & & & & \\
\hline Duration of illness, $y$ & $4.9(2.7)$ & II.5 (3.2) & & & 108 & & & $<0.001$ \\
\hline-0.5 & 0 & 0 & & & & & & \\
\hline $0.6-1.0$ & 10 & 0 & & & & & & \\
\hline I.I-3.0 & 22 & 0 & & & & & & \\
\hline $3.1-6.0$ & 19 & 2 & & & & & & \\
\hline $6.1-$ & 24 & 13 & & & & & & \\
\hline Duration of treatment, $y$ & $3.4(2.5)$ & $6.2(4.0)$ & & & 346 & & & 0.019 \\
\hline-0.5 & 7 & 1 & & & & & & \\
\hline $0.6-1.0$ & 18 & 2 & & & & & & \\
\hline $1.1-3.0$ & 19 & 3 & & & & & & \\
\hline $3.1-6.0$ & 18 & 1 & & & & & & \\
\hline $6.1-$ & 13 & 8 & & & & & & \\
\hline
\end{tabular}

Abbreviations: BD, bipolar disorder; H, Kruskal-Wallis nonparametric multiple sample test; HAMD, Hamilton's Rating Scale for Depression; HC, healthy controls; MDD, major depressive disorder; U, Mann-Whitney nonparametric 2-sample test.

The database for the healthy controls contained data from 40 males and 40 females each aged 54-86 (mean $70.2 \pm 7.3$ ) years. Their z-score maps were displayed in color on seismic tomographic and standardized surface-rendering images (Figure 1). ${ }^{24}$

The sACC (parts of Brodmann areas 24, 32, and 33) on the surface-rendering images was set as the area of interest to identify atrophy, and colored areas in the left and right sACCs were determined as atrophic lesions. For diagnosis of Alzheimer's disease, areas are displayed in color when the $\mathrm{z}$-score is $\geq 2$, ie, a threshold or risk rate of $P<0.05$ in comparison with the database for healthy controls. In this study, receiver operating characteristic analysis was done to confirm the validity of the score in MDD diagnosis. The presence or absence of atrophy in the sACC was compared based on the presence or absence of a color-displayed area on the z-score map for the patients with MDD or bipolar disorder and for the healthy subjects. We then investigated VSRAD plus and VSRAD advance for their performance as diagnostic tools in MDD.

\section{Results}

Receiver operating characteristic analysis for the VSRAD advance confirmed the validity of a z-score of 2.0 as the threshold for color display on the z-score maps. No significant difference was noted in the area under the curve on receiver operating characteristic analysis between the VSRAD plus and the VSRAD advance (Figure 2). Diagnoses using the VSRAD advance identified atrophy in the sACC in 68 (63 on the left side and 65 on the right side) of the 75 patients with MDD, with no significant difference between the left and right sACCs. Bilateral atrophy of the SACC was noted in one of the 15 patients with bipolar disorder. Atrophy was noted in four (four each on the left and right sides) of the 30 healthy subjects, with no difference between the left and right sACCs. Using the VSRAD plus, atrophy was identified in 62 of the 75 patients with MDD, six of the 15 patients with bipolar disorder, and five of the 30 healthy subjects. No lateralization was noted in atrophy of the sACC in any group. Significant differences were noted in the sACC atrophy rate detected using either the VSRAD advance or VSRAD plus between the MDD and bipolar disorder groups and between the MDD and healthy subject groups (Table 2). Moreover, with the VSRAD advance, no correlation was noted between atrophy of the sACC and disease severity, duration of illness, or duration of treatment. Regarding the accuracy of MDD diagnosis in comparison with healthy subjects, the VSRAD advance had a sensitivity of $90.7 \%$, specificity of $86.7 \%$, 


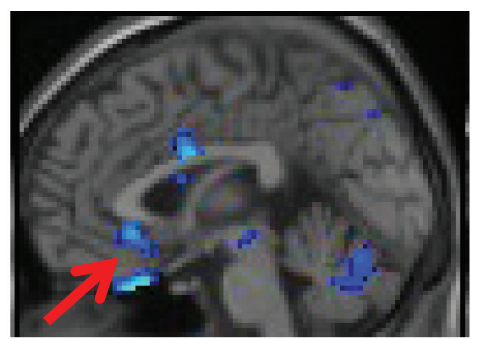

Sagittal

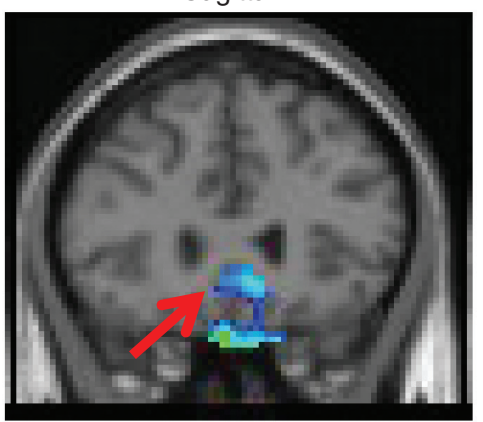

Coronal

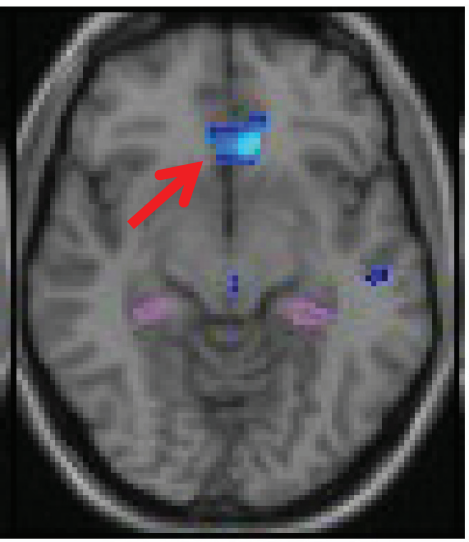

Axial

2.0

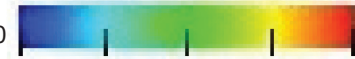

z-score
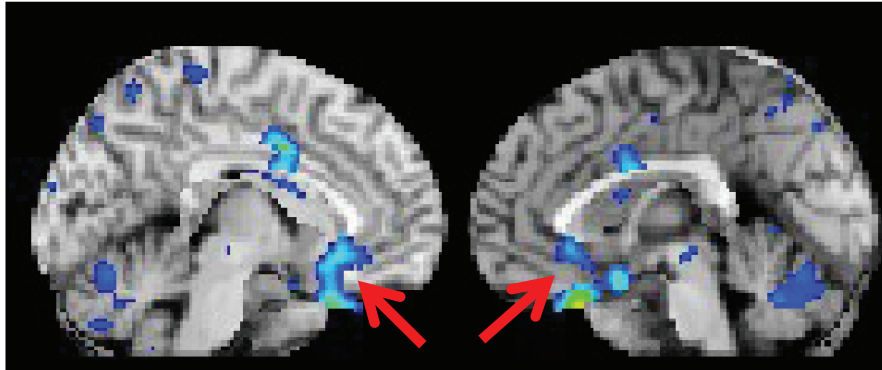

Left medial

Right medial

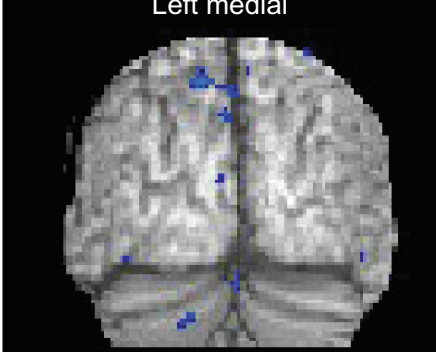

Posterior

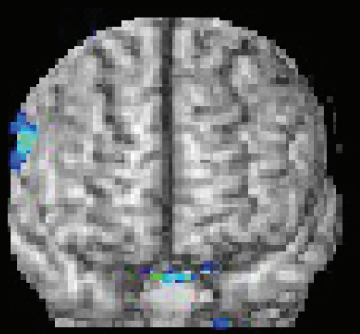

Anterior

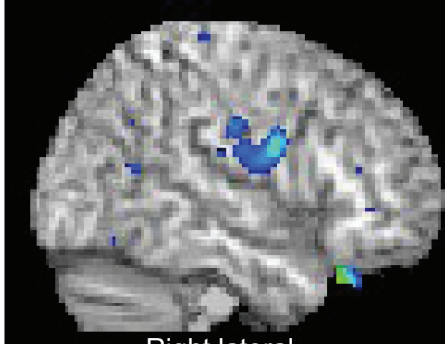

Right lateral
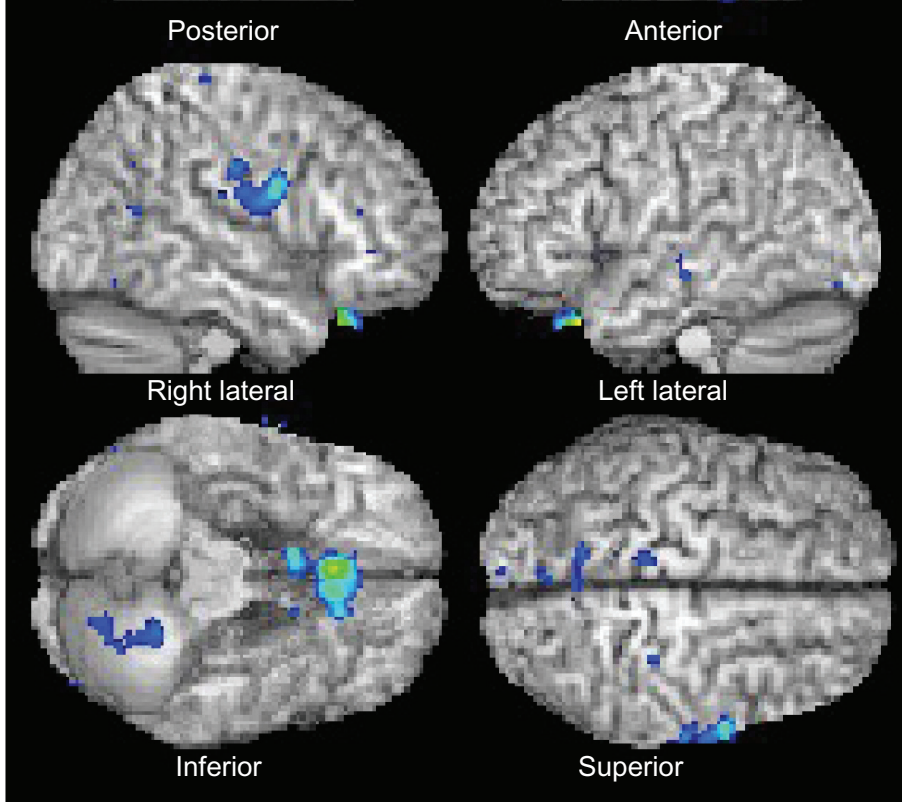

Figure I z-score maps of a 62-year-old woman with major depressive disorder. Colored areas indicating a z-score of $\geq 2$ are overlaid as significantly atrophied regions of the sACC on tomographic and cortical surface images presented on a standardized magnetic resonance imaging template (arrows).

accuracy of $89.5 \%$, a positive predictive value (PPV) of $94.4 \%$, and a negative predictive value (NPV) of $78.8 \%$, which was markedly higher compared with the VSRAD plus, which showed a sensitivity, specificity, accuracy, PPV, and NPV of $82.7 \%, 83.3 \%, 82.9 \%, 92.5 \%$, and $65.8 \%$, respectively (Table 3).

The shapes of the colored areas superimposed on the z-score maps were classified into four patterns. The first was a dot pattern displayed on the subcallosal area of the sACC (parts of Brodmann areas 24, 32, and 33). This pattern was noted in ten of the 68 patients with MDD who presented with atrophy of the sACC. The second one, a linear pattern that presents itself on the subcallosal area of the SACC, was identified in 14 patients. The third one, a massive pattern on the sACC (including the subcallosal area), was observed in 24 patients. The fourth pattern, which is usually observed in the medial prefrontal area, including the SACC, was noted in 20 patients (Table 4 and Figure 3).

In summary, in all cases in which atrophy of the sACC was noted, atrophy of the subcallosal area of the sACC was identified. These results suggest that the identification of atrophy of the subcallosal area of the sACC is important. 


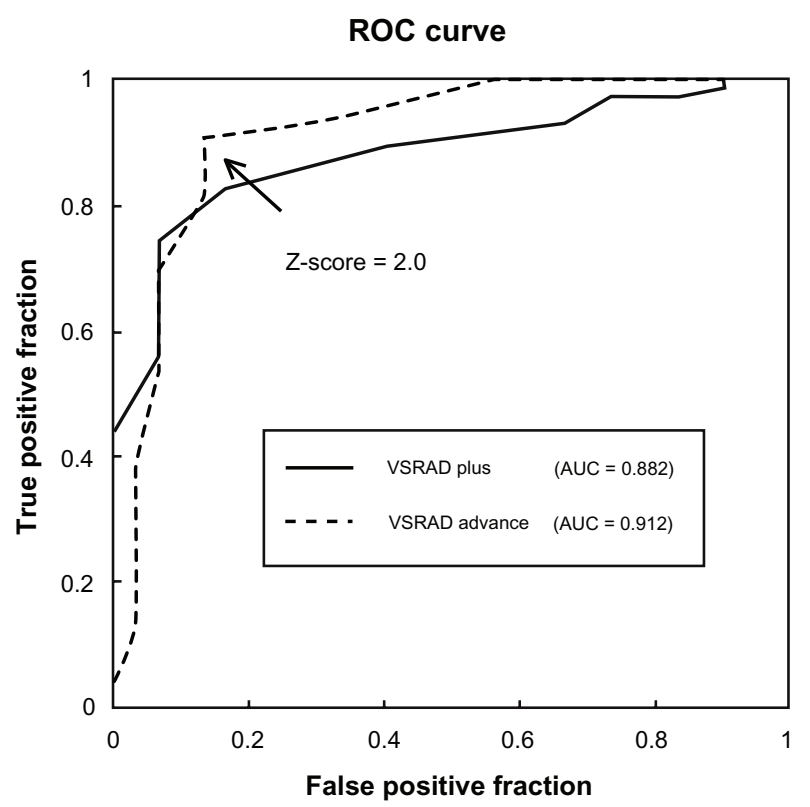

Figure $2 \mathrm{~A}$ ROC curve was drawn to distinguish patients with major depressive disorder and healthy subjects based on the z-score in the subgenual anterior cingulate cortex set as the threshold on VSRAD plus and VSRAD advance. Abbreviation: ROC, receiver operating characteristic.

\section{Discussion}

Although there have been several studies conducted on atrophy of the subgenual cingulate cortex in MDD, few of them have been used in the clinical setting. ${ }^{17,18,25,26}$ In previous research, we have focused on the VSRAD plus, an early diagnostic support system for Alzheimer's disease, which allowed us to identify regional atrophy in the brain easily, based on images obtained from MRI scans. ${ }^{4}$ We showed that identification of atrophy of the sACC (through a visual method) served as an effective auxiliary means of diagnosis for MDD.

In the present study, we used the VSRAD advance, a voxel-based morphometry-based software for the automatic

Table 2 Atrophy of sACC of VSRAD plus vs VSRAD advance

\begin{tabular}{|c|c|c|c|c|c|}
\hline Application & \multicolumn{2}{|c|}{ VSRAD plus } & \multicolumn{3}{|c|}{ VSRAD advance } \\
\hline $\operatorname{MDD}(n=75)$ & $62(83 \%)$ & & & 68 (9I\%) & \\
\hline Right & $57(76 \%)$ & & & $65(87 \%)$ & \\
\hline Left & 54 (72\%) & $P<0.001$ & NS [ & $63(84 \%)$ & $P<0.001$ \\
\hline $\mathrm{BD}(\mathrm{n}=15)$ & $6(40 \%)$ & & & I (7\%) & \\
\hline Right & $5(33 \%)$ & $P<0.001$ & & I I (7\%) & $P<0.001$ \\
\hline Left & NS $L_{5}(33 \%)$ & & & L I (7\%) & \\
\hline $\mathrm{HC}(\mathrm{n}=30)$ & $5(17 \%)$ & & & $4(13 \%)$ & \\
\hline Right & $5(17 \%)$ & & & $4(13 \%)$ & \\
\hline Left & $L_{3}(10 \%)$ & & & $L_{4}(13 \%)$ & \\
\hline
\end{tabular}

Abbreviations: BD, bipolar disorder; HC, healthy controls; MDD, major depressive disorder; NS, no significant difference; sACC, subgenual anterior cingulate cortex; VSRAD, Voxel-based Specific Regional analysis system for Alzheimer's Disease.
Table 3 Diagnostic proportion of MDD vs HC

\begin{tabular}{lll}
\hline & $\begin{array}{l}\text { VSRAD plus } \\
\text { (\%) }\end{array}$ & $\begin{array}{l}\text { VSRAD advance } \\
\text { (\%) }\end{array}$ \\
\hline Sensitivity & 82.7 & 90.7 \\
Specificity & 83.3 & 86.7 \\
Accuracy & 82.9 & 89.5 \\
PPV & 92.5 & 94.4 \\
NPV & 65.8 & 78.8 \\
\hline
\end{tabular}

Abbreviations: HC, healthy controls; MDD, major depressive disorder; NPV, negative predictive value; PPV, positive predictive value; VSRAD, Voxel-based Specific Regional analysis system for Alzheimer's Disease.

detection of specific atrophy in early Alzheimer's disease upgraded with the SPM8 and DARTEL, for distinguishing patients with MDD from healthy subjects. The sensitivity was $90.7 \%$, and the specificity, accuracy, PPV, and NPV were $86.7 \%, 89.5 \%, 94.4 \%$, and $78.8 \%$, respectively. The accuracy of the VSRAD advance was markedly higher compared with that of the VSRAD plus. This was presumably attributed to the newly adopted algorithms, which allowed the SPM8 to isolate gray matter images more efficiently, as well as the more accurate anatomical standardization onto templates by DARTEL. ${ }^{27,28}$ Given that the number of healthy subjects was limited at the outpatient clinics, further investigation involving more healthy volunteers is awaited for accuracy of the PPV and NPV. Nevertheless, the results are still useful for comparison of the plus and advance methods.

In this study, there were massive and spreading forms of atrophy in the sACC, and dot and linear forms of atrophy were particularly noted in the subcallosal area of the sACC. In all cases in which atrophy of the SACC was noted, atrophy of the subcallosal area of the sACC was identified. In 48 of the 68 cases, as the threshold for the z-score maps was lowered, colored areas first started to appear in the subcallosal area of the sACC, and atrophy was extensive in this area, suggesting that the presence or absence of atrophy of the subcallosal area of the sACC has a significant meaning in the diagnosis of MDD.

Table 4 The shapes of colored areas on cortical surface of the standardized MRI template

\begin{tabular}{ll}
\hline Shape & $\mathbf{n = 6 8}$ \\
\hline Dot & 10 \\
Line & 14 \\
Massive & 24 \\
Wide-spread & 20 \\
\hline
\end{tabular}




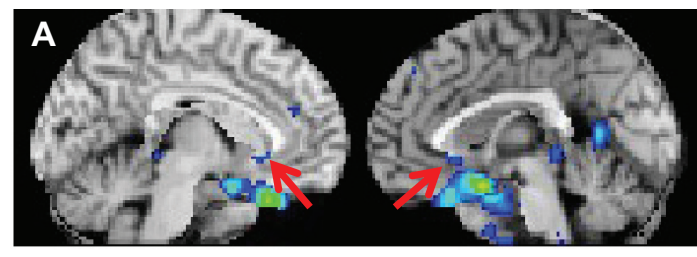

Left medial

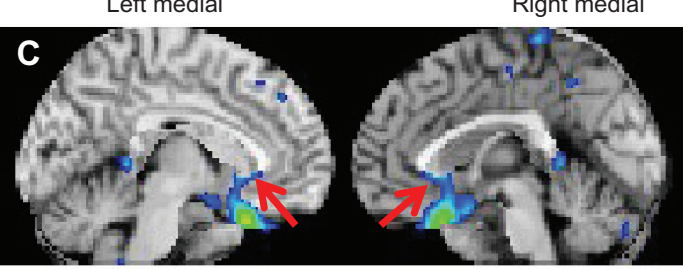

Left medial

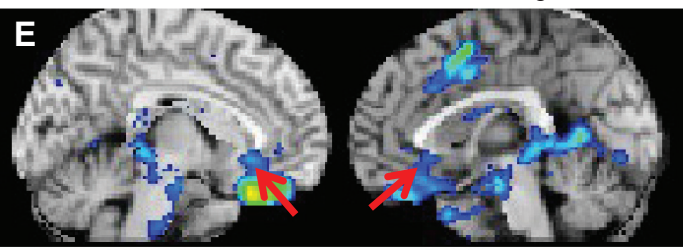

Left medial

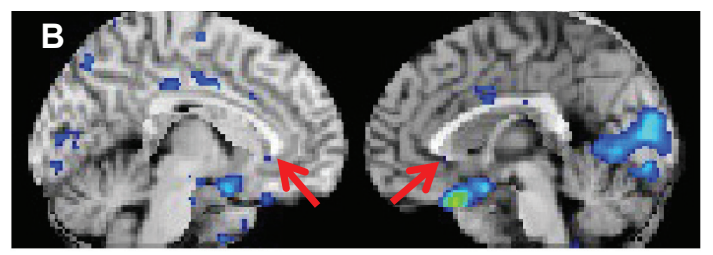

Left medial

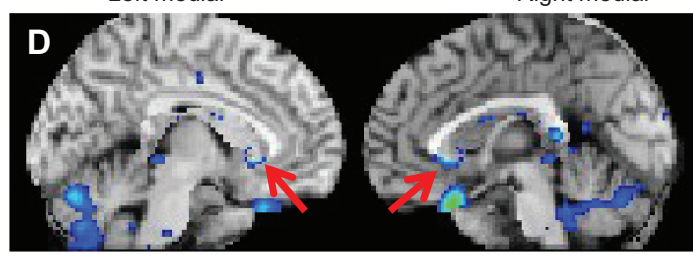

Left medial

Right medial

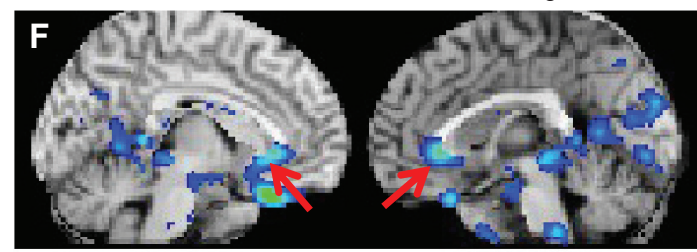

Left medial

Right medial

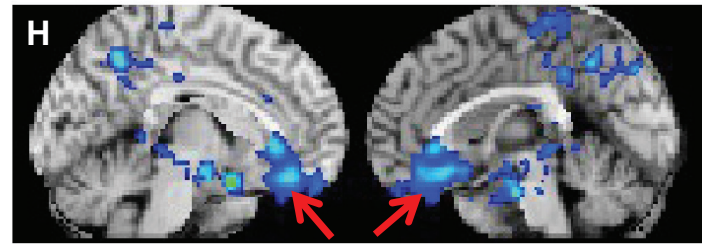

Left medial

Right medial
Right medial

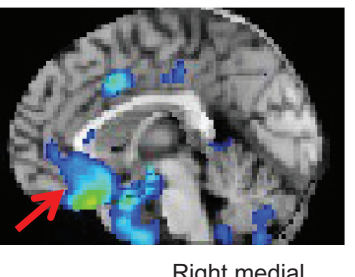

6.0

Figure 3 z-score maps of patients with major depressive disorder (A) for a 70-year-old woman and (B) for a 7I-year-old woman. Colored areas in dot-like form are overlaid on the cortical surface of the subgenual anterior cingulate cortex displayed on a standardized MRI template (arrows). (C) Images for a 57-year-old woman and (D) for a 59-year-old woman. Colored areas in linear form are overlaid on the subgenual anterior cingulate cortex (arrows). (E) Image for a 62-year-old woman and (F) for a 68-year-old woman. Colored areas in massive form are overlaid on the subgenual anterior cingulate cortex (arrows). (G) Image for a 72 -year-old woman and (H) for a 58-year-old woman. Colored areas in spreading form are overlaid on the subgenual prefrontal cortex including the subgenual anterior cingulate cortex (arrows).

The results of the present study suggest that the VSRAD advance, providing more accurate diagnosis including sensitivity, accuracy, and NPV, is effective as an auxiliary diagnostic method for MDD with a high degree of accuracy. When using this method in clinical practice, careful attention should be paid to the following points. There was only a slight increase in specificity and PPV, which would indicate that some healthy subjects also have atrophy of the sACC. In other words, atrophy of the sACC is also noted in those other than patients with MDD. What then does atrophy of the sACC mean? Previous investigators reported atrophy of the subgenual cingulate cortex and amygdala in carriers of the $s$ allele for serotonin transporter polymorphism. ${ }^{29}$ Another study has reported that a larger percentage of carriers of $s$ allele polymorphism who had undergone four or more stressful life events over the past five years developed depression compared with $l / l$-type carriers. ${ }^{30}$ There is also a report that no significant differences were noted in cingulate volumes between currently depressed and remitted patients. ${ }^{31}$ There was no marked difference in the level of atrophy in nine MDD patients with atrophy of the sACC re-examined after a period of 302-546 (mean $476 \pm 55.5$ ) days.

According to these reports, atrophy of the sACC is genetically determined, and it indicates a carrier of an $s$ allele polymorphism or vulnerability to depression. Atrophy of the subgenual cingulate cortex observed in young patients with MDD may add to its evidence. ${ }^{25}$ Therefore, this method serves to diagnose vulnerability to depression rather than MDD itself. When atrophy of the sACC is not identified in a patient complaining of depressive symptoms following an MRI examination in daily clinical practice, we can determine that the patient will be responsive to antidepressant medication or consider the possibility of other diseases. ${ }^{4}$ Nevertheless, the fact that atrophy of the sACC is not noted can be valuable clinical information in addition to medical interviews and observation. 
In order to improve the objectivity of the method, it is necessary to specify the volume of interest and calculate the mean z-score and rate of atrophy within the area as well as the ratio of atrophy to that in the whole brain. It is also necessary to collect data from a large number of health care facilities to determine acceptable margins among different institutions and equipment.

With improvement designed to specify automatically the volume of interest and calculate the mean volume of interest z-score and rate of atrophy within the area as well as the ratio to atrophy in the whole brain, the auxiliary diagnostic method would be even simpler and more effective.

\section{Conclusion}

The present study examined the effectiveness of identification of atrophy in the sACC with VSRAD advance, upgraded with SPM8 and DARTEL, as a diagnostic method for MDD. It was suggested that the simple auxiliary method for the diagnosis of MDD was effective for distinguishing patients with MDD from healthy subjects, and the sensitivity, specificity, accuracy, and PPV values were $90.7 \%, 86.7 \%, 89.5 \%$, and $94.4 \%$, respectively. In patients with MDD, atrophy was identified in localized areas of the subcallosal area in the sACC (parts of Brodmann areas 24, 32, and 33), which suggests that identification of atrophy in the subcallosal area is important for the diagnosis of MDD.

\section{Acknowledgments}

The authors are indebted to Professor Scott Fraser for his valuable comments on the manuscript and constructive suggestions regarding this paper. We are also grateful to Takako Kuniyoshi from the Nanto Clinic for her encouragement and administrative support.

\section{Disclosure}

The authors report no conflicts of interest in this work.

\section{References}

1. Hirata Y, Matsuda H, Nemoto K, et al. Voxel-based morphometry to discriminate early Alzheimer's disease from controls. Neurosci Lett. 2005;382(3):269-274.

2. Matsuda H. Role of neuroimaging in Alzheimer's disease, with emphasis on brain perfusion SPECT. J Nucl Med. 2007;48(8):1289-1300.

3. Matsuda $H$. The role of neuroimaging in mild cognitive impairment. Neuropathology. 2007;27(6):570-577.

4. Niida R, Niida A, Motomura M, Uechi A. Diagnosis of depression by MRI scans with the use of VSRAD - a promising auxiliary means of diagnosis: a report of 10 years research. Int J Gen Med. 2011;4: 377-387.

5. MervaaIa E, Föhr J, Könönen M, et al. Quantitative MRI of the hippocampus and amygdala in severe depression. Psychol Med. 2000; 30(1):117-125.
6. Sheline YI, Gado MH, Kraemer HC. Untreated depression and hippocampal volume loss. Am J Psychiatry. 2003;160(8):1516-1518.

7. Lange C, Irle E. Enlarged amygdala volume and reduced hippocampal volume in young women with major depression. Psychol Med. 2004; 34(6):1059-1064.

8. Frodl T, Schaub A, Banac S, et al. Reduced hippocampal volume correlates with executive dysfunctioning in major depression. J Psychiatry Neurosci. 2006;31(5):316-323.

9. Campbell S, MacQueen G. An update on regional brain volume differences associated with mood disorders. Curr Opin Psychiatry. 2006;19(1):25-33.

10. Yucel K, McKinnon MC, Chahal R, et al. Anterior cingulate volumes in never-treated patients with major depressive disorder. Neuropsychopharmacology. 2008;33(13):3157-3163.

11. Sheline YI, Gado MH, Price JL. Amygdala core nuclei volumes are decreased in recurrent major depression. Neuroreport. 1998;9(9): 2023-2028.

12. Yoshikawa E, Matsuoka Y, Yamasue H, et al. Prefrontal cortex and amygdala volume in first minor or major depressive episode after cancer diagnosis. Biol Psychiatry. 2006;59(8):707-712.

13. Wagner G, Koch K, Schachtzabel C, Reichenbach JR, Sauer H, Schlösser RG. Enhanced rostral anterior cingulate cortex activation during cognitive control is related to orbitofrontal volume reduction in unipolar depression. J Psychiatry Neurosci. 2008;33(3):199-208.

14. Hickie IB, Naismith SL, Ward PB, et al. Serotonin transporter gene status predicts caudate nucleus but not amygdala or hippocampal volumes in older persons with major depression. J Affect Disord. 2007; 98(1-2):137-142.

15. Bremner JD, Vythilingam M, Vermetten E, et al. Reduced volume of orbitofrontal cortex in major depression. Biol Psychiatry. 2002;51(4):273-279.

16. Taki Y, Kinomura S, Awata S, et al. Male elderly subthreshold depression patients have smaller volume of medial part of prefrontal cortex and precentral gyrus compared with age-matched normal subjects: a voxel-based morphometry. J Affect Disord. 2005;88(3): 313-320.

17. Drevets WC, Price JL, Simpson JR, et al. Subgenual prefrontal cortex abnormalities in mood disorders. Nature. 1997;386(6627):824-827.

18. Hastings RS, Parsey RV, Oquendo MA, Orango V, Mann JJ. Volumetric analysis of the prefrontal cortex, amygdala, and hippocampus in major depression. Neuropsychopharmacology. 2004;29(5):952-959.

19. Tang Y, Wang F, Xie G, et al. Reduced ventral anterior cingulate and amygdala volumes in medication-naive females with major depressive disorder: a voxel-based morphometric magnetic resonance imaging study. Psychiatry Res. 2007;156(1):83-86.

20. Frodl T, Jäger M, Born C, et al. Anterior cingulate cortex does not differ between patients with major depression and healthy controls, but relatively large anterior cingulate cortex predicts a good clinical course. Psychiatry Res. 2008;163(1):76-83.

21. Yuan Y, Zhu W, Zhang Z, et al. Regional gray matter changes are associated with cognitive deficits in remitted geriatric depression: an optimized voxel-based morphometry study. Biol Psychiatry. 2008; 64(6):541-544.

22. Drevets WC, Savitz J, Trimble M. The subgenual anterior cingulate cortex in mood disorders. CNS Spectr. 2008;13(8):663-681.

23. Koolschijn PC, van Haren NE, Lensve1t-Mulders GJ, Hulshoff Pol HE, Kahn RS. Brain volume abnormalities in major depressive disorder: a meta-analysis of magnetic resonance imaging studies. Hum Brain Mapp. 2009;30(11):3719-3735.

24. Matsuda H, Mizumura S, Nemoto K, et al. Automatic voxel-based morphometry of structural MRI by SPM8 plus diffeomorphic anatomic registration through exponentiated lie algebra improves the diagnosis of probable Alzheimer disease. AJNR Am J Neuroradiol. 2012;33(6): 1109-1114,

25. van Tol MJ, van der Wee NJ, van den Heuvel OA, et al. Regional brain volume in depression and anxiety disorders. Arch Gen Psychiatry. 2010; 67(10):1002-1011. 
26. Amico F, Meisenzahl E, Koutsouleris N, Reiser M, Möller HJ, Frodl T. Structural MRI correlates for vulnerability and resilience to major depressive disorder. J Psychiatry Neurosci. 2011;36(1): 15-22.

27. Ashburner J. A fast diffeomorphic image registration algorithm. Neuroimage. 2007;38(1):95-113.

28. Pereira JM, Xiong L, Acosta-Cabronero J, Pengas G, Williams GB, Nestor PJ. Registration accuracy for VBM studies varies according to region and degenerative disease grouping. Neuroimage. 2010;49(3): 2205-2015.
29. Pezawas L, Meyer-Lindenberg A, Drabant EM, et al. 5-HTTLPR polymorphism impacts human cingulate-amygdala interactions: a genetic susceptibility mechanism for depression. Nat Neurosci. 2005;8(6): 828-834.

30. Dannlowski U, Ohrmann P, Bauer J, et al. Serotonergic genes modulate amygdala activity in major depression. Genes Brain Behav. 2007;6(7): 672-676.

31. Caspi A, Sugden K, Moffitt TE, et al. Influence of life stress on depression: moderation by a polymorphism in the 5-HTT gene. Science. 2003;301(5631):386-389.

\section{Publish your work in this journal}

The International Journal of General Medicine is an international, peer-reviewed open-access journal that focuses on general and internal medicine, pathogenesis, epidemiology, diagnosis, monitoring and treatment protocols. The journal is characterized by the rapid reporting of reviews, original research and clinical studies across all disease areas.
A key focus is the elucidation of disease processes and management protocols resulting in improved outcomes for the patient.The manuscript management system is completely online and includes a very quick and fair peer-review system. Visit http://www.dovepress.com/ testimonials.php to read real quotes from published authors.

Submit your manuscript here: http://www.dovepress.com/international-journal-of-general-medicine-journal 